\title{
Review of Topical Lightening Agents
}

\section{Shuba Dharmana*}

Evangelist Le'Jeune Medspa, Bangalore, India

\begin{abstract}
Hyperpigmentation occurs when there is an increase in melanin content in the skin. It can be localised i.e. limited to a certain area or it can be diffuse i.e. occurring all over the body. Localised form of hyperpigmentation can happen as a result of injury or inflammation from conditions such as Acne, contact dermatitis, lupus etc known as post inflammatory hyperpigmentation or Melasma. Diffuse generally results from a systemic disease, drug or neoplasm. Localised hyperpigmentation like $\mathrm{PIH}$ and melasma can lead to a lot of psychological distress with skin type IV and above being particularly susceptible. It is not only important to find the cause of pigmentation in order to arrest further progression but it is also important to treat the pigmented lesions. Further prevention should advocate the use of daily sun protection with sunscreen agents and other sun avoidance measures.
\end{abstract}

Keywords: Melasma; Hyperpigmentation; Melanin

\section{Introduction}

Hyperpigmentation occurs when there is an increase in melanin content in the skin. It can be localised i.e. limited to a certain area or it can be diffuse i.e. occurring all over the body. Localised form of hyperpigmentation can happen as a result of injury or inflammation from conditions such as Acne, contact dermatitis, lupus etc known as post inflammatory hyperpigmentation or Melasma. Diffuse generally results from a systemic disease, drug or neoplasm. Localised hyperpigmentation like PIH and melasma can lead to a lot of psychological distress [1] with skin type IV and above being particularly susceptible [2]. It is not only important to find the cause of pigmentation in order to arrest further progression but it is also important to treat the pigmented lesions. Further prevention should advocate the use of daily sun protection with sunscreen agents and other sun avoidance measures.

\section{Topical Agents}

Number of topical agents is used to treat PIH. The most commonly used agent is hydroquinone between 2 to $10 \%$ though 2 to $4 \%$ of hydroquinone alone or with of tretinoin 0.05 to $0.1 \%$ is used [3]. Hydroquinone is a hydroxyphenolic chemical classically used in melasma treatment.

\section{Hydroquinone}

Hydroquinone is one of the important agents when treating PIH. It is a phenol compound which inhibits tyrosinase, thereby reducing the conversion of dihydroxyphenylalanine (DOPA) to melanin [4]. In the US, hydroquinone is available over the counter (OTC) at $2 \%$ and in a prescription strength from $3 \%$ to $10 \%$, although hydroquinone is typically used at $2-4 \%$ concentrations $[5,6]$.

Daily concomitant use of a broad-spectrum sunscreen can also be beneficial as sun exposure can hinder the effects of hydroquinone, leading to repigmentation. Antioxidants such as $0.5 \%$ ascorbic acid (vitamin C) and retinoid as well as a-hydroxy acids are used as additives to increase penetration and enhance efficacy. Cook- Bolden and Hamilton [7] conducted a 12-week, open-label study of microencapsulated $4 \%$ hydroquinone and $0.15 \%$ retinol with antioxidants in the treatment of 21 patients (17 PIH, 4 melasma). Significant decreases in lesion size, pigmentation, and disease severity were noted as early as 4 weeks after initiating treatment and remained significantly decreased throughout the study (all $\mathrm{p}<0.032$ ). The majority of patients $(63 \%)$ experienced either marked improvement (75\% overall improvement) or complete clearing ( $95 \%$ or greater overall improvement), and reflectance spectrophotometer readings showed statistically significant reductions in melanin content as early as week 4 . Similar results were obtained in a previous study [8]. The $5 \%$ concentration of hydroquinone may also be compounded with $0.5 \%$ ascorbic acid in a low-potency corticosteroid base to increase penetration and reduce irritation, respectively. An improvement in the hyperpigmentation may be noticeable after 4 weeks of therapy but the optimal effect is typically achieved after 6-10 weeks of therapy [9]. Prolonged daily use of $4-5 \%$ hydroquinone can lead to a high incidence of irritant reactions particularly when combined with retinoic acid [9].

A triple combination cream in a stabilized formulation, containing $4 \%$ hydroquinone, $0.05 \%$ tretinoin, and $0.01 \%$ fluocinolone acetonide, has been demonstrated to be both safe and effective in the treatment of moderate to severe melasma [6,9-11] and has also been successfully used in the treatment of PIH, although formal clinical studies are needed.

In 1982, the US FDA originally proposed that OTC hydroquinone at $1.5-2 \%$ concentrations was generally safe and effective. However, in 2006, the FDA released a statement proposing a ban on all OTC hydroquinone agents and a requirement for any currently marketed hydroquinone product to submit New Drug Application (NDA) or be withdrawn from the market [12]. This change in position was based on rodent studies, which suggested that oral hydroquinone may be a carcinogen [12]. The FDA has yet to make a final ruling. In light of the controversy surrounding the use of topical hydroquinone, clinicians are looking to other products with depigmenting effects. Mequinol, tretinoin monotherapy, and azelaic acid have also been proven to be effective in the treatment of PIH.

\section{Mequinol}

Mequinol (4-hydroxyanisole) is a derivative of hydroquinone but may cause less skin irritation than its parent compound. Mequinol may involve in competitive inhibition of tyrosinase thereby inhibiting melanin formation [13]. An open-label, split face, non-

${ }^{*}$ Corresponding author: Dr. Shuba Dharmana, Le'Jeune Medspa, 52, Vittal Mallya Road, 1st floor, Bangalore, 560 001, India, Tel: +91 9845104890; E-mail: sdharmana@gmail.com

Received: September 05, 2014; Accepted: October 20, 2014; Published: October 24, 2014

Citation: Dharmana S (2014) Review of Topical Lightening Agents. Pigmentary Disorders 1:145. doi: 10.4172/JPD.1000145

Copyright: () 2014 Dharmana S. The terms of the Creative Commons Attribution License, which permits unrestricted use, distribution, and reproduction in any medium, provided the original author and source are credited. 
inferiority, comparison study of $2 \%$ mequinol $/ 0.01 \%$ tretinoin versus $4 \%$ hydroquinone cream was conducted in 61 patients with mild to moderate facial PIH [14]. Patients applied $2 \%$ mequinol $/ 0.01 \%$ tretinoin to one side of the face and $4 \%$ hydroquinone to the contra lateral side for 12 weeks. The mequinol/tretinoin solution was found to be non-inferior to the hydroquinone cream in the treatment of PIH as $81 \%$ of mequinol/tretinoin-treated and $85 \%$ of hydroquinone-treated patient's experienced clinical success.

\section{Retinoids}

Tretinoin (all-trans retinoic acid), a vitamin A analog, used to treat hyperpigmentation of photo aged skin, post inflammatory hyperpigmentation, and melasma. Tretinoin $0.1 \%$ has been used to successfully treat melasma in Black patients; improvements of up to $73 \%$ were seen after 40 weeks of treatment [5]. Erythema and peeling in the area of application are adverse effects of tretinoin 0.05 to $0.1 \%$; post inflammatory hyperpigmentation can also occur.

Concentrations range from $0.01 \%$ to $0.1 \%$. However, irritant dermatitis can be a frequent adverse effect, and caution should be used when prescribing higher concentrations of tretinoin in darker skin types, since the irritation can lead to further PIH. To determine the safety and efficacy of $0.1 \%$ tretinoin in the treatment of $\mathrm{PIH}$, a randomized, double-blind, vehicle-controlled clinical trial was conducted in 54 Black patients [5]. Patients applied either tretinoin or vehicle cream to the face, arms, or both areas daily for 40 weeks. At the study endpoint, the PIH lesions of the tretinoin-treated group were significantly lighter than those of the vehicle-treated group as assessed by clinical $(\mathrm{p}<0.001)$ and colorimetric $(\mathrm{p}=0.05)$ examinations. However, it is important to note that patients treated with tretinoin experienced minimal lightening of normal skin and 50\% of these patients also developed retinoid dermatitis. It is possible to reduce this irritation by reducing dose and titrating to higher doses gradually or by using cream based formulations [15] or even diluting it with a moisturizer.

Other retinoid compounds are also available including isotretinoin, adapalene, and tazarotene. Isotretinoin is available in both oral and topical formulations and is widely used in the treatment of acne.

\section{Azelaic acid}

Topical azelaic acid 15 to $20 \%$ can be as efficacious as hydroquinone, but is less of an irritant.

Azelaic acid is a dicarboxylic acid isolated from Pityrosporum ovale, the organism responsible for pityriasis versia color [9].

Studies have shown that $20 \%$ azelaic acid cream produces significantly greater decreases in pigmentary intensity than the vehicle [16] and, when combined with $15-20 \%$ glycolic acid, is as effective as $4 \%$ hydroquinone in the treatment of facial hyperpigmentation including melasma and PIH [17]. Azelaic acid is generally well tolerated with only mild adverse effects reported, such as pruritus, transient erythema, scaling, and irritation, which disappear in a few weeks [18].

\section{Kojic Acid}

Kojic acid has recently been used as a $2 \%$ cream, alone or in combination with glycolic acid or hydroquinone, due to its inhibitory action on tyrosinase. In a comparative study in Chinese women with epidermal melasma, half of the face was treated with kojic acid $2 \%$ gel plus glycolic acid $10 \%$ and hydroquinone $2 \%$, and the other half of the face with just glycolic acid $10 \%$ and hydroquinone $2 \%$ [19]. Better results were obtained on the half of the face treated with the cream including kojic acid.

A common adverse effect with kojic acid use is contact dermatitis $[20,21]$. Although kojic acid had been widely used in skin care products in Japan, more recent clinical studies conducted there have demonstrated its highly sensitizing potential [22]. Kojic acid has also been recently banned from the Japanese market over mutagenicity concerns.

\section{Arbutin}

Arbutin works by inhibiting tyrosinase activity as well as melanosome maturation, and its efficacy is concentration dependent but higher concentrations can lead to a paradoxical hyperpigmentation [23]. a-Arbutin and deoxyarbutin are both synthetic forms with greater tyrosinase inhibition than the original arbutin $[24,25]$. Clinical studies have demonstrated that $3 \%$ deoxyarbutin is effective in lightening solar lentigines in light-skinned patients [26]; however, there are no data on its efficacy for PIH.

\section{Niacinamide}

Niacinamide is the water-soluble, physiologically active derivative of niacin (vitamin B3). Topical niacinamide has been used in the treatment of acne but studies now suggest that there may be a role for niacinamide in the treatment of pigmentary disorders. Hakozaki et al. [27] showed through in vitro studies that niacinamide decreases melanosome transfer by $35-68 \%$ without inhibiting tyrosinase activity or cell proliferation. Research also suggests that niacinamide may decrease melanogenesis by interfering with the interaction between keratinocytes and melanocytes but not through a directly inhibitory effect on melanocytes. Two to five percent niacinamide has proven to be an effective lightening agent for patients with melasma or UV induced hyperpigmentation [27-29]; however, studies are needed to determine its safety and efficacy in the treatment of PIH.

\section{N-Acetyl Glucosamine}

Multiple, double-blind, controlled, clinical trials demonstrated the clear benefit of $2 \% \mathrm{~N}$-acetyl glucosamine in the treatment of hyperpigmentation secondary to sun exposure $[28,30]$ but none secondary to inflammatory causes.

\section{Ascorbic Acid (Vitamin C)}

Ascorbic acid is a depigmenting agent that suppresses melanin synthesis by its antioxidant properties, Ascorbic acid is not a very effective skin lightening agent when used as monotherapy; however, $5-10 \%$ ascorbic acid is still frequently formulated in combination with other depigmenting agents such as hydroquinone given its excellent safety profile [31]. Further studies are needed to determine the utility of ascorbic acid in the management of PIH. However, ascorbic acid has shown some efficacy as combination therapy for photo aging and melasma.

\section{Licorice Extract}

Licorice extract is a very common component of depigmenting cosmetic products sold worldwide. There are several active agents in licorice extract including glabridin and licochalcone $\mathrm{A}$, which have both been shown to inhibit tyrosinase activity $[32,33]$. In addition, glabridin exhibits anti-inflammatory properties. Liquiritin, another active ingredient of licorice extract, does not inhibit tyrosinase activity but causes depigmentation by melanin dispersion [34]. There are very few clinical studies with licorice extract; however, liquiritin $1 \mathrm{~g}$ /day has 
been shown to be effective treatment for melasma, although no patients with PIH were included in the study.

\section{Soy}

Soy extracts are commonly used in cosmetic skin moisturizers for their skin-lightening properties [31] and a manufacturer's study has demonstrated improvement of certain dyschromias including PIH with the use of a daily SPF 15 soy moisturizer. Whole soy extract has also shown some efficacy in the treatment of PIH secondary to acne when formulated with salicylic acid and retinol.

\section{Chemical Peels}

One of the most common indications for chemical peeling is dyschromias such as PIH. Chemical peels used alone or in combination with lightening agents can be very efficacious. Typically, superficial chemical peels have been studied as therapy for PIH. These peels can be used in all skin types, and are commonly used in patients with darker skin tones because they are generally well tolerated with proven results. Care should be taken in selecting the specific chemical peel to avoid possible irritation, which can worsen PIH. Patients should also practice sun avoidance and wear sunscreen to avoid PIH after having received a chemical peel.

Chemical peeling with glycolic acid utilizes strengths ranging from $20 \%$ to $70 \%$ and requires neutralization with water or sodium bicarbonate to terminate the peel. Burns et al. [35] conducted a study of 16 African American patients with facial PIH in which the control group was treated with $2 \%$ hydroquinone $/ 10 \%$ glycolic acid gel and $0.05 \%$ tretinoin and the peel group was treated with the same topical regimen plus a series of six glycolic acid peels (50-68\%) at 3-week intervals. Significant clinical improvement was noted compared to baseline in the peel group using the Hyperpigmentation Area and Severity Index (HASI) $(\mathrm{p}<0.02)$; however, the difference between the two treatment groups was close to but did not reach statistical significance. The peel group also experienced a more rapid and greater improvement by colorimetric analysis but results were of borderline significance $(\mathrm{p}<0.09)$. The authors also reported a greater lightening of normal pigmentation in the peel group.

Superficial salicylic acid peels typically utilize strengths ranging from $20 \%$ to $30 \%$. A clinical study was conducted using salicylic acid peels in 24 Korean patients with PIH secondary to acne [36]. Salicylic acid (30\%) peels were performed every 2 weeks for 3 months. Although the level of lightness from baseline to the first post-peel period was significant $(p<0.02)$ by colorimetric analysis, final levels did not reach statistical significance. However, colorimetric analysis did show a highly significant reduction in erythema $(\mathrm{p}<0.0001)$, and clinical improvement was noted in greasiness, dryness, scaliness, and erythema at the study endpoint.

\section{Laser Therapy}

There are a number of different lasers and light sources currently available that can also be effective for PIH. Due to the wide absorption spectrum of melanin, which ranges from 250 to $1200 \mathrm{~nm}$, melanin can be targeted specifically by all visible light and near-infrared dermatologic lasers currently in use [37].

Lasers with shorter wavelengths are not optimal for treatment in darker-skinned patients since the individual's normal epidermal melanin is also targeted at these wavelengths. The absorption coefficient of melanin decreases exponentially as the wavelength increases [38]. Therefore, lasers generating wavelengths that are less efficiently absorbed by endogenous melanin, such as the $1064 \mathrm{~nm}$ QS neodymium: yttrium-aluminum-garnet (Nd:YAG) laser can provide a greater margin of safety while still allowing the laser surgeon to achieve satisfactory results in darker-skinned individuals.

\section{Emerging Therapies}

As there is more demand for new depigmenting agents, A recent, randomized, double-blind, vehicle-controlled clinical trial showed that $2 \%$ undecylenoyl phenylalanine effectively treats solar lentigines [39], and a published case-report explains how topical 5\% methimazole was successfully used to treat PIH secondary to a chemical burn [40]. Dioic acid has been compared with hydroquinone in the treatment of melasma, showing it to be a possible treatment alternative [41], and aloesin has been shown in studies to suppress pigmentation induced by UV radiation [42]. Other agents that require further research and development include 4-(1-phenylethyl)1,3-benzenediol, paper mulberry, ellagic acid, quinolines, piperlonguminine, luteolin, and calycosin [20].

\section{Conclusion}

Hyperpigmented disorders of the skin pose a significant medical and esthetic problem for individuals with any skin complexion. A variety of topical and systemic agents have been employed in the treatment of these disorders. Many studies are conducted and being conducting however more studies are needed to elucidate the exact role of most of the agents used for depigmentation. As the cosmetic industry is growing faster, more agents are expected to be discovered. Simultaneously market/consumers are looking for alternatives for prescribed treatments for skin lightening and inclining towards natural/ neutral agents.

\section{References}

1. Taylor A, Pawaskar M, Taylor SL, Balkrishnan R, Feldman SR (2008) Prevalence of pigmentary disorders and their impact on quality of life: a prospective cohort study. J Cosmet Dermatol 7: 164-168.

2. Rossi AM, Perez MI (2011) Treatment of hyperpigmentation. Facial Plast Surg Clin North Am 19: 313-324.

3. Pérez-Bernal A Muñoz-Pérez MA (2000) Management of Facial Hyperpigmentation. Am J Clin Dermatol 1: 261-268.

4. Palumbo A, d'Ischia M, Misuraca G, Prota G (1991) Mechanism of inhibition of melanogenesis by hydroquinone. Biochim Biophys Acta 1073: 85-90.

5. Kimbrough-Green CK, Griffiths CE, Finkel LJ, Hamilton TA, Bulengo-Ransby SM, et al. (1994) Topical retinoic acid (tretinoin) for melasma in black patients. A vehicle-controlled clinical trial. Arch Dermatol 130: 727-733.

6. Lacz NL, Vafaie J, Kihiczak NI, Schwartz RA (2004) Postinflammatory hyperpigmentation: a common but troubling condition. Int J Dermatol 43: 362365 .

7. Cook-Bolden FE, Hamilton SF (2008) An open-label study of the efficacy and tolerability of microencapsulated hydroquinone $4 \%$ and retinol $0.15 \%$ with antioxidants for the treatment of hyperpigmentation. Cutis $81: 365-371$.

8. Grimes PE (2004) A microsponge formulation of hydroquinone $4 \%$ and retino $0.15 \%$ in the treatment of melasma and postinflammatory hyperpigmentation. Cutis 74: 362-368

9. Jimbow K, Minamitsuji Y (2001) Topical therapies for melasma and disorders of hyperpigmentation. Dermatol Ther 14: 35-45.

10. Kligman AM, Willis I (1975) A new formula for depigmenting human skin. Arch Dermatol 111: 40-48.

11. Chan R, Park KC, Lee MH, Lee ES, Chang SE, et al. (2008) A randomized controlled trial of the efficacy and safety of a fixed triple combination (fluocinolone acetonide $0.01 \%$, hydroquinone $4 \%$, tretinoin $0.05 \%$ ) compared with hydroquinone $4 \%$ cream in Asian patients with moderate to severe melasma. Br J Dermatol 159: 697-703. 
12. Levitt J (2007) The safety of hydroquinone: a dermatologist's response to the 2006 Federal Register. J Am Acad Dermatol 57: 854-872.

13. Draelos ZD (2006) The combination of $2 \%$ 4-hydroxyanisole (mequinol) and $0.01 \%$ tretinoin effectively improves the appearance of solar lentigines in ethnic groups. J Cosmet Dermatol 5: 239-244.

14. Taylor SC, Callender VD (2006) A multicenter, 12-week, phase 3b trial: a combination solution of mequinol $2 \%$ /tretinoin $0.01 \%$ vs hydroquinone $4 \%$ cream in the treatment of mild to moderate postinflammatory hyperpigmentation. J Am Acad Dermatol 54: AB194.

15. Callender VD (2004) Acne in ethnic skin: special considerations for therapy. Dermatol Ther 17: 184-195.

16. Lowe NJ, Rizk D, Grimes P, Billips M, Pincus S (1998) Azelaic acid $20 \%$ cream in the treatment of facial hyperpigmentation in darker-skinned patients. Clin Ther 20: 945-959.

17. Kakita LS, Lowe NJ (1998) Azelaic acid and glycolic acid combination therapy for facial hyperpigmentation in darker-skinned patients: a clinical comparison with hydroquinone. Clin Ther 20: 960-970.

18. Ortonne JP, Passeron T (2005) Melanin pigmentary disorders: treatment update. Dermatol Clin 23: 209-226.

19. Lim JT (1999) Treatment of melasma using kojic acid in a gel containing hydroquinone and glycolic acid. Dermatol Surg 25: 282-284.

20. Grimes PE (2009) Management of hyperpigmentation in darker racial ethnic groups. Semin Cutan Med Surg 28: 77-85.

21. Serra-Baldrich E, Tribó MJ, Camarasa JG (1998) Allergic contact dermatitis from kojic acid. Contact Dermatitis 39: 86-87.

22. Nakagawa M, Kawai K, Kawai K (1995) Contact allergy to kojic acid in skin care products. Contact Dermatitis 32: 9-13.

23. Maeda K, Fukuda M (1996) Arbutin: mechanism of its depigmenting action in human melanocyte culture. J Pharmacol Exp Ther 276: 765-769.

24. Funayama M1, Arakawa H, Yamamoto R, Nishino T, Shin T, et al. (1995) Effects of alpha- and beta-arbutin on activity of tyrosinases from mushroom and mouse melanoma. Biosci Biotechnol Biochem 59: 143-144.

25. Boissy RE, Visscher M, DeLong MA (2005) DeoxyArbutin: a novel reversible tyrosinase inhibitor with effective in vivo skin lightening potency. Exp Dermatol 14: 601-608

26. Boissy RE1, Visscher M, DeLong MA (2005) DeoxyArbutin: a novel reversible tyrosinase inhibitor with effective in vivo skin lightening potency. Exp Dermato 14: 601-608.

27. Hakozaki T, Minwalla L, Zhuang J, Chhoa M, Matsubara A, et al. (2002) The effect of niacinamide on reducing cutaneous pigmentation and suppression of melanosome transfer. Br J Dermatol 147: 20-31.
28. Kimball AB, Kaczvinsky JR, Li J, Robinson LR, Matts PJ, et al. (2010) Reduction in the appearance of facial hyperpigmentation after use of moisturizers with a combination of topical niacinamide and $\mathrm{N}$-acetyl glucosamine: results of a randomized, doubleblind, vehicle-controlled trial. $\mathrm{Br}$ J Dermatol 162: 435-441.

29. Bissett DL, Miyamoto K, Sun P, Li J, Berge CA (2004) Topical niacinamide reduces yellowing, wrinkling, red blotchiness, and hyperpigmented spots in aging facial skin. Int J Cosmet Sci 26: 231-238.

30. Bissett DL, Robinson LR, Raleigh PS, Miyamoto K, Hakozaki T, et al. (2007) Reduction in the appearance of facial hyperpigmentation by topical $\mathrm{N}$-acety glucosamine. J Cosmet Dermatol 6: 20-26.

31. Draelos ZD (2007) Skin lightening preparations and the hydroquinone controversy. Dermatol Ther 20: 308-313.

32. Fu B, Li H, Wang X, Lee FS, Cui S (2005) Isolation and identification of flavonoids in licorice and a study of their inhibitory effects on tyrosinase. J Agric Food Chem 53: 7408-7414.

33. Yokota T, Nishio H, Kubota Y, Mizoguchi M (1998) The inhibitory effect of glabridin from licorice extracts on melanogenesis and inflammation. Pigment Cell Res 11: 355-361.

34. Amer M, Metwalli M (2000) Topical liquiritin improves melasma. Int J Dermato 39: 299-301.

35. Burns RL, Prevost-Blank PL, Lawry MA, Lawry TB, Faria DT, et al. (1997) Glycolic acid peels for postinflammatory hyperpigmentation in black patients. A comparative study. Dermatol Surg 23: 171-174.

36. Ahn HH, Kim IH (2006) Whitening effect of salicylic acid peels in Asian patients Dermatol Surg 32: 372-375.

37. Tanzi EL, Alster TS (2004) Cutaneous laser surgery in darker skin phototypes. Cutis 73: $21-24,27-30$

38. Alster TS, Tanzi EL (2003) Laser surgery in dark skin. Skinmed 2: 80-85.

39. Katoulis AC1, Alevizou A, Bozi E, Makris M, Zafeiraki A, et al. (2010) A randomized, double-blind, vehiclecontrolled study of a preparation containing undecylenoyl phenylalanine $2 \%$ in the treatment of solar lentigines. Clin Exp Dermatol 35: 473-476.

40. Kasraee B, Handjani F, Parhizgar A, Omrani GR, Fallahi MR, et al. (2005) Topical methimazole as a new treatment for postinflammatory hyperpigmentation: report of the first case. Dermatology 211: 360-362.

41. Tirado-Sánchez A, Santamaría-Román A, Ponce-Olivera RM (2009) Efficacy of dioic acid compared with hydroquinone in the treatment of melasma. Int $J$ Dermatol 48: 893-895

42. Choi S, Lee SK, Kim JE, Chung MH, Park YI (2002) Aloesin inhibits hyperpigmentation induced by UV radiation. Clin Exp Dermatol 27: 513-515. 